\title{
Das American Friends Service Committee. Humanitäre Identität und die Politics of Service
}

„Was vergangen, kehrt nicht wieder, aber ging es leuchtend nieder, leuchtet's lange noch zurück." Professor Adalbert Czerny bemühte den deutschen Romantikdichter Karl August Förster, als er 1922 den Quäkern des US-amerikanischen American Friends Service Committee (AFSC) für ihre soeben zu Ende gegangene Kinderspeisungsaktion in Deutschland dankte. Die Quäker, so Czerny, Ordinarius für Kinder- und Jugendheilkunde an der Berliner Charité und einer der Begründer der modernen Pädiatrie, hätten ein Werk in Gang gebracht, das „an Geschlossenheit und Ehrlichkeit alle anderen Versuche, dem deutschen Volke in seiner Not $\mathrm{zu}$ helfen“, übertroffen habe und das darüber hinaus, was seine „wissenschaftlichen Methoden“ betraf, als leuchtendes Vorbild in Erinnerung bleiben werde. ${ }^{1}$

Czernys Lob war kein Einzelfall im Europa der Zwischenkriegsjahre. So wie er kamen Millionen von Menschen unter anderem in Frankreich, Deutschland, Russland, Österreich und Spanien in Berührung mit den Hilfsaktionen des 1917 gegründeten AFSC, der zentralen Hilfsorganisation der US-amerikanischen Quäker, ${ }^{2}$ die gleichermaßen als Vertreterin eines universellen religiös-humanitären Impetus sowie amerikanischer Großzügigkeit und Fortschrittlichkeit auftrat. An vielen Orten leuchteten diese Aktivitäten in der Tat noch lange nach, etwa im weitverbreiteten Gebrauch des Begriffs „Quäkerspeisungen“ in Deutschland. Der historische Rückblick lässt vor allem ein Erstaunen lebendig werden, das schon die Zeitgenossen erfüllte: Es ist das Erstaunen über die scheinbare Omnipräsenz und den Einfluss dieser kleinen protestantischen Sekte, die weltweit zu Anfang des 20. Jahrhunderts nicht mehr als etwa 250.000 Mitglieder hatte.

1 Adalbert Czernys Rede in: Archives of the American Friends Service Committee, Philadelphia (im Folgenden: AFSCA), Foreign Service, Country: Germany 1921.

2 Zur Nomenklatur: In der folgenden Arbeit verwende ich die Begriffe „Quäker“ und „Friends“ als Synonyme, ebenso wie „Quäkertum“ und Religious Society of Friends als Beschreibungen der Gesamtheit der Quäker/Friends in einem Land. Ursprünglich als ein Spottname verwendet, der sich auf die vermeintlich ekstatische religiöse Praxis (to quake = sich schütteln, zittern) bezog, hat sich „Quäker“ bereits im 18. Jahrhundert als Fremd- und Eigenbezeichnung durchgesetzt. Dagegen ist „Religiöse Gemeinschaft der Freunde“ (Religious Society of Friends) der offizielle Name der Glaubensgemeinschaft geblieben, ebenso wie die Bezeichnung der Anhänger als „Friends“. Daraus ergibt sich eine Vielzahl von Ableitungen beider Bezeichnungen, die im Verlauf der folgenden Arbeit immer wieder auftauchen werden. Quaker relief, Quaker embassies, Quaker college auf der einen Seite, Friends Ambulance Unit, Friends War Victim Relief Committee, Friends Service Committee etc. auf der anderen.

Ә OpenAccess. (C) 2022 Daniel Maul, publiziert von De Gruyter. (cc) BY-NC-ND Dieses Werk ist lizenziert unter einer Creative Commons Namensnennung - 4.0 International Lizenz.

https://doi.org/10.1515/9783110675788-002 
Es ist zuvorderst die Frage nach der Triebfeder der Hilfe, ihrer Motive und der mit ihr verbundenen Ziele, die das folgende Buch umtreibt. Die übergeordnete Frage ist damit mindestens so alt wie die Geschichte humanitärer Hilfe selbst: Warum helfen Menschen anderen Menschen über die Grenzen der eigenen Familie, des eigenen sozialen Umfelds, des eigenen nationalen Zusammenhangs hinweg? Wenn humanitäre Hilfe ein Medium darstellt, das Menschen über lokale, nationale, ja kontinentale Grenzen hinweg miteinander in Beziehung setzt: Welche Kräfte treiben diese Hilfe an? Welche Rolle spielen politische, kulturelle, ethnische, wirtschaftliche, psychologische und andere Faktoren? Welches Gewicht wird diesen Faktoren jeweils zugeschrieben - in der Selbstwahrnehmung der Organisatoren der Hilfe sowie in der Außenwahrnehmung durch die Empfänger der Hilfe, durch die Politik, die Öffentlichkeit, die Medien?

Die Männer und Frauen des AFSC erscheinen bei der Beantwortung dieser Fragen in vielerlei Hinsicht als ein ideales Studienobjekt. Ihre religiöse Verwurzelung innerhalb der Gemeinschaft der Quäker sowie ihr gleichzeitiges Auftreten als Vertreterinnen und Vertreter einer dezidiert amerikanischen Hilfsorganisation erscheinen als die Hauptprägungskräfte dessen, was ich im Folgenden als humanitäre Identität des AFSC beschreiben möchte. Als eine ihrem Wesen nach transnational orientierte und insbesondere transatlantisch vernetzte Gruppe von Akteuren mit einem gleichzeitig deutlich nationalen Profil eignet sich das AFSC darüber hinaus besonders gut, die der humanitären Hilfe im Untersuchungszeitraum inhärenten Spannungen zu diskutieren. Die Gründung des AFSC im Jahr des amerikanischen Kriegseintritts 1917 und der Aufstieg des Komitees zu einer professionellen Hilfsorganisation mit Einsätzen an einer Reihe von europäischen Schauplätzen erlaubt einen tiefen Einblick in die Zwischenkriegsjahre als eine Phase des humanitären Aufbruchs. Das Netz der Beziehungen, welches die Quäker in diesen Jahren mit einer Vielzahl von Akteuren verknüpfte, reicht von Hilfsagenturen wie dem Amerikanischen Roten Kreuz (American Red Cross - ARC) oder dem Jewish Joint Distribution Committee (JDC) bis ins amerikanische State Department, von Großbritannien über Frankreich, Deutschland und die Sowjetunion bis nach Spanien und schließlich bis in die Korridore des Genfer Völkerbundes. Am Beispiel und durch die Augen des AFSC werden auf diese Weise entstehende Mechanismen internationaler Hilfe sowie die Ansätze eines globalen humanitären Regimes sichtbar.

Zwei den humanitären Aktivitäten der Quäker innewohnende Grundspannungen stellen dabei die Achsen der Untersuchung dar und machen diese gleichzeitig anschlussfähig an die breitere Forschung zur Geschichte humanitärer Hilfe im 20. Jahrhundert. Es ist zum einen die Spannung zwischen dem universellen Anspruch einer über die Grenzen der USA hinaus vernetzten und agierenden Gruppe von Menschen einerseits und deren tiefer Verankerung in einem 
nationalen Kontext andererseits. Das AFSC baute bei seiner Arbeit im Europa der Zwischenkriegszeit auf lange etablierte transnationale, im Wesentlichen transatlantische Verbindungen auf. Zur selben Zeit behielt das Komitee über den gesamten Beobachtungszeitraum seinen amerikanischen Charakter bei: Seine Öffentlichkeitsarbeit und seine Spendenkampagnen waren ausschließlich auf ein amerikanisches Publikum ausgerichtet, und seine Aktionen waren ohne Ausnahme in ein breiteres humanitär-diplomatisches Netz amerikanischer Institutionen eingebunden, vom Militär über andere amerikanische Hilfsagenturen bis in die Aussenpolitik. Der permanente Aushandlungsprozess, bei dem das AFSC versuchte seine Position in diesem Spannungsfeld zu bestimmen, war einer der Hauptantriebe bei der Entwicklung der Quäkerhilfe, die sich parallel und in enger Beziehung zum Aufstieg der USA zur „humanitarian superpower“ vollzog. ${ }^{3}$

Im gleichen Sinne muss auch die zweite Grundspannung, welche die Geschichte des AFSC bestimmte, im größeren Kontext betrachtet werden: Diese Spannung ergab sich aus dem Anspruch, Notleidenden die nach allgemein gültigen Standards bestmögliche Hilfe zu leisten, und dem gleichzeitigen Ziel, bei dieser Hilfe als Quäker erkennbar zu bleiben. Die Anfänge einer Quäkerhilfsorganisation in den USA waren Teil einer Entwicklung, in der humanitäre Hilfe einen präzedenzlosen Bedeutungsgewinn erzielte, der sich mit den Begriffen der Institutionalisierung und Professionalisierung beschreiben lässt. Das AFSC entstand zu einem Zeitpunkt, an dem sich in zuvor nicht gekanntem Ausmaß ein „humanitärer Markt“ entwickelte, auf dem unterschiedliche Gruppen um Aufmerksamkeit und Ressourcen kämpften. Die Notwendigkeit, sich auf diesem Markt zu behaupten und das eigene Profil als Hilfsorganisation zu schärfen, stellte die Quäker vor ein schwer aufzulösendes Dilemma, vor allem da eine solchermaßen am Markt und einer „moral economy“ der Hilfe orientierte Tätigkeit viele unterschiedliche und oftmals widerstreitende Dinge gleichzeitig bedeuten konnte, je nachdem ob das Quäkerspezifische, mithin die tieferliegende Botschaft der Hilfe, oder aber im Gegensatz dazu eine humanitäre Professionalität in den Vordergrund trat. Beides miteinander zu versöhnen - to do the right job oder to do the job right - stellte das AFSC vor eine schwierige Aufgabe und war zur selben Zeit eine Triebfeder, die die institutionelle Entwicklung des Komitees bestimmte. ${ }^{4}$

3 Daniel Maul, „The Rise of a Humanitarian Superpower: American NGOs and International Relief, 1917-1945, in: Miguel Bandeira Jerónimo and José Pedro Monteiro (Hg.) Internationalism, Imperialism and the Formation of the Contemporary World. The Pasts of the Present. (Basingstoke: Palgrave Macmillan, 2018).

4 Zum humanitären Markt und den Eigenheiten der humanitären „moral economy“ siehe Norbert Götz, Georgina Brewis und Steffen Werther, Humanitarianism in the Modern World: The Moral Economy of Famine Relief, (New York: Cambridge University Press, 2020); Rebecca Gill, Calcula- 


\section{Humanitarismus als Geschichte}

Die Geschichte humanitärer Hilfe und des humanitären Sektors in der Zwischenkriegszeit ist das übergeordnete Thema dieses Buches. Sie hat sich in den vergangenen Jahren zu einem der facettenreichsten und fruchtbarsten Bereiche der historischen Forschung entwickelt. ${ }^{5}$ Ein Grund hierfür liegt im noch immer nicht voll ausgeschöpften Potenzial humanitärer Hilfe als Indikator für eine Fülle von anderen Themen vom Lokalen hin zum Globalen. Humanitäre Hilfe setzt Menschen auf vielfältige Weise innerhalb und über nationale Grenzen hinweg in Beziehung zueinander. Sie dient auf den unterschiedlichsten Ebenen, von lokalen Spendenaktionen bis auf die Bühnen der Weltpolitik, als ein Medium, durch das Kontakte und Ideen entstehen, durch das Hierarchien konstruiert und reflektiert, gefestigt und in Frage gestellt werden. Sie erzeugt in jedem Fall wirkungsmächtige Bilder, die unsere Wahrnehmung von anderen und nicht zuletzt von uns selbst prägen.

Allein die jüngste Forschung liefert eine Fülle unterschiedlicher Blickwinkel auf das Thema, sei es zu geschlechterspezifischen Fragen, ${ }^{6}$ zur Rolle der Medien ${ }^{7}$, $\mathrm{zu}$ bestimmten humanitären Techniken und ihrer wissenschaftlichen Einbindung ${ }^{8}$ oder der moral economy der Hilfe. ${ }^{9}$ Humanitäre Hilfe dient als Lackmustest bei der Untersuchung unterschiedlicher historischer Entwicklungen, etwa zum

ting Compassion: Humanity and Relief in War, Britain 1870-1914 (Manchester: Manchester University Press, 2013); Michelle Tusan, „The Business of Relief Work: A Victorian Quaker in Constantinople and Her Circle“, in: Victorian Studies 51, 4 (2009), 633-663.

5 Zum state of the art siehe die Konversation zwischen einigen der herausragenden Forscherinnen und Forscher auf dem Feld in: „History and Humanitarianism: A Conversation“, in: Past and Present 241, 1 (2018). Einen guten Überblick liefern Silvia Salvatici, A History of Humanitarianism, 1755-1989: In the Name of Others, Humanitarianism Series (Manchester: Manchester University Press, 2019); Michael Barnett, Empire of Humanity: A History of Humanitarianism (Ithaca: Cornell University Press, 2011); Johannes Paulmann, „Conjunctures in the History of Humanitarian Aid During the Twentieth Century“, in: Humanity 4, 2 (2013), 215-238.

6 Esther Möller, Johannes Paulmann und Katharina Stornig (Hg.), Gendering Global Humanitarianism in the Twentieth Century: Practice, Politics and the Power of Representation (London: Palgrave Macmillan, 2020).

7 Johannes Paulmann, Humanitarianism \& Media: 1900 to the Present, New German Historical Perspectives 9 (New York: Berghahn Books, 2019); Heide Fehrenbach und Davide Rodogno, Humanitarian Photography: A History, Human Rights in History (New York: Cambridge University Press, 2015).

8 Joël Glasman, Humanitarianism and the Quantification of Human Needs: Minimal Humanity, Routledge Humanitarian Studies (London/New York: Routledge, 2020).

9 Götz, Brewis und Werther, Humanitarianism in the Modern World: The Moral Economy of Famine Relief. 
Internationalismus und dem Entstehen internationaler Strukturen der Hilfe ${ }^{10}$ oder der wachsenden Bedeutung zivilgesellschaftlicher Gruppen auf nationaler oder internationaler Ebene. ${ }^{11}$ Humanitäre Organisationen erscheinen als Akteure vor dem Hintergrund der großen weltpolitischen Umwälzungen des 20. Jahrhunderts, der beiden Weltkriege, des Kalten Krieges und der Dekolonisierung. ${ }^{12}$

Das vorliegende Buch schließt unmittelbar an diese Forschungen an. Seine Annäherung an die Geschichte der Quäkerhilfe während der Zwischenkriegszeit ist dabei beides: Sie steht pars pro toto für zentrale Entwicklungen des humanitären Sektors einerseits, die sich in den Hilfsaktionen des AFSC spiegeln. Sie behandelt anderseits diese Aktionen als eigenständigen Beitrag einer Gruppe von Akteuren, deren spezifische „Positionalität“, deren Traditionen, sozialer Hintergrund, Erfahrungen und andere gruppenspezifische Attribute ihre Handlungen vorprägten. Bei der Analyse des Beitrags der amerikanischen Quäker zur Entstehung eines potenziell global orientierten humanitären Sektors bietet Daniel Laquas doppeldeutige Metapher von der „humanitarian cloud“ einen guten Ausgangspunkt. ${ }^{13}$ Gleich einer Wolke dient der Sektor als verlässlicher Seismograf klimatischer Veränderungen, sprich: wechselnder historischer Kontexte; seine Konturen selbst bleiben dabei jedoch jederzeit fließend. Zur selben Zeit lässt sich der Sektor in Analogie zum cloud computing als lernender Mechanismus, als „Datenwolke“ verstehen, die Informationen, sprich: Erfahrungen speichert und den Teilnehmern jederzeit zur Verfügung stellt. Die Quäker erscheinen dabei als eine der vielen unterschiedlichen Teile der humanitarian cloud der Zwischenkriegszeit, neben religiösen und säkularen, evangelikalen und nichtmissionarischen, politisch progressiven und reaktionären Gruppen, die ihre Erfahrungen in die Wolke einspeisen und umgekehrt aus ihr Informationen beziehen. Diese Erfahrungen zeigen sich auf den unterschiedlichsten Feldern: bei der Durchführung von Spendenkampagnen und bei der Öffentlichkeitsarbeit, in der eigenen Annäherung an humanitäre Prinzipien wie Neutralität oder Unparteilichkeit oder bei der Anwendung wissenschaftlicher Erkenntnisse aus der Ernährungswissenschaft oder der Logistik.

10 Jessica Reinisch, „Internationalism in Relief: The Birth (and Death) of UNRRA“, in: Past and Present 210, Supplement 6 (2011), 258-289.

11 Matthew Hilton et al., The Politics of Expertise: How NGOs Shaped Modern Britain (Oxford: Oxford University Press, 2013).

12 Siehe etwa die Beiträge in: Emily Baughan und Bronwen Everill (Hg.), Special Issue: Empire and Humanitarianism, 40 (Journal of Imperial and Commonwealth History, 2012).

13 Daniel Laqua, „Inside the Humanitarian Cloud: Causes and Motivations to Help Friends and Strangers“, in: Journal of Modern European History 12, 2 (2014), 175-186. 
Die Quäker zeigen sich dabei gleichzeitig, in Michael Barnetts Worten, als archetypische „Alchemisten“, die über den technischen Aspekt ihrer Hilfe gleichsam höhere und langfristigere Ziele verfolgten. ${ }^{14}$ Ihre Aktionen waren in der Tat niemals, wie die folgende Arbeit zeigen wird, von dem Anspruch zu trennen, durch das Medium der Hilfe eine Reihe von höheren, transzendenten Zielen zu verwirklichen, die von religiösen Inhalten im engeren Sinn bis hin zu praktischer Friedens- und Versöhnungsarbeit reichen konnten. Damit eignen sich die Quäker besonders gut für eine Diskussion der Motive, humanitäre Hilfe zu leisten. Mein Argument im Folgenden wird lauten, dass gerade die Spannung zwischen professioneller Hilfsarbeit und dem Ziel, diese Hilfe mit spezifischen Inhalten zu verknüpfen, einen der Hauptmotoren der Entwicklung der Quäkerhilfe darstellt. Gleichzeitig erscheint diese Spannung, in der Terminologie des AFSC zwischen message work und relief work, anschlussfähig an eine Grundspannung, die den humanitären Sektor als Ganzen kennzeichnet; die Erfahrung und wiederum der Umgang damit sind in diesem Sinn, um das Bild noch einmal zu bemühen, der gewichtigste Beitrag der Quäker zur humanitarian cloud.

Trotz der geschilderten Allgegenwart der Quäker auf den europäischen Krisenschauplätzen und ihrer engen Verbindungen zur breiteren humanitären Bewegung der Zwischenkriegszeit hat das AFSC bislang außerhalb eines kleineren Kreises von Quaker historians noch kaum Aufmerksamkeit gefunden. Gregory Barnes Buch, das im Auftrag des AFSC anlässlich dessen hundertsten Jahrestages 2017 herausgegeben wurde, ist bislang die einzige verfügbare Gesamtdarstellung, die ein nützliche Orientierung gibt und nicht zuletzt im Rahmen der vorliegenden Arbeit zum besseren Verständnis der internen Entwicklungen des AFSC und seiner Arbeit in den USA selbst hilfreich war. ${ }^{15}$ Daneben haben eine Reihe teils über den US-amerikanischen Kontext hinausreichender Werke die Quäkerhilfe als Teil der breiteren Entwicklung der Society of Friends behandelt. ${ }^{16}$ Nicht viel anders ist

14 Barnett, Empire of Humanity: A History of Humanitarianism.

15 Gregory A. Barnes, A Centennial History of the American Friends Service Committee (Philadelphia: FriendsPress, 2016).

16 Pink Dandelion, An Introduction to Quakerism (Cambridge: Cambridge University Press, 2007); Stephen W. Angell und Pink Dandelion (Hg.), The Cambridge Companion to Quakerism (Cambridge: Cambridge University Press, 2018); Stephen W. Angell und John Connell, „Quakers in North America“, in: Stephen W. Angell und Pink Dandelion (Hg.), The Cambridge Companion to Quakerism (Cambridge: Cambridge University Press, 2018), 161-178; Ilana Feldman, „The Quaker Way: Ethical Labor and Humanitarian Relief“, in: American Ethnologist 34, 4 (2007), 689-705; Thomas D. Hamm und Isaac Barnes May, „Conflict and Transformation, 1808-1920“, in: Stephen W. Angell und Pink Dandelion (Hg.), The Cambridge Companion to Quakerism (Cambridge: Cambridge University Press, 2018), 31-49; Timothy Burdick und Pink Dandelion, „Global Quakerism 1920 - 2015“, in: Stephen W. Angell und Pink Dandelion (Hg.), The Cambridge Companion 
die Lage für einzelne Hilfseinsätze während der Zwischenkriegszeit, die in der Regel isoliert voneinander und stets ohne Bezugnahme auf den breiteren humanitären Kontext behandelt werden. Gerade mit Blick auf die zwei quantitativ und qualitativ bedeutendsten Hilfseinsätze des AFSC in den 1920er Jahren, in Deutschland von 1919 bis 1923 und in der Sowjetunion von 1921 bis 1923, bestehen weiterhin beträchtliche Leerstellen. ${ }^{17}$ Durch Forschungen zur Eigenart der Quäkerhilfe und umgekehrt ihre Einordung in ihren breiteren historischen Kontext eröffnet sich auf diese Weise ein noch weithin unbestelltes Feld.

Der Befund zur relativen Stille um die Quäkerhilfe im 20. Jahrhundert muss umso mehr verwundern, als Literatur, die sich mit der longue durée der Quäkerhilfe befasst, auf eine lange Tradition zurückblickt. Beiträge aus der „neuen Kulturgeschichte“, die sich mit den Ursprüngen des Humanitarismus und menschenrechtlichen Denkens befasst, sehen ein mit der Aufklärung verknüpftes, neuartiges „Gefühlsregime“ und die damit verbundene gewachsene „Sensibilität“ für menschliches Leiden als treibende Kraft hinter der Ausbreitung humanitärer Aktivitäten an der Schwelle zum 19. Jahrhundert. ${ }^{18}$ Andere interpretieren die AntiSklaverei-Bewegung und gleichgerichtete Unternehmungen, die darauf zielten das Leid von distant sufferers zu mindern, als Ausdruck eines neuartigen rationalen Bewusstseins um die Kausalität menschlichen Handelns, in der sich zuallererst, so die These, ein neues, im Kern kapitalistisches Denken manifestierte. Wie Thomas Haskell es ausdrückt: Je mehr Menschen glaubten, ihr ökonomisches

to Quakerism (Cambridge: Cambridge University Press, 2018), 49-66; Für Großbritannien siehe auch John Ormerod Greenwood, Quaker Encounters Vol. 1: Friends and Relief (York: William Sessions Limited, 1975); Thomas C. Kennedy, British Quakerism 1860-1920: The Transformation of a Religious Community (Oxford: Oxford University Press, 2001).

$17 \mathrm{Zu}$ Deutschland nach dem Ersten Weltkrieg, das nach wie vor eine der auffälligesten Forschungslücken darstellt siehe etwa Guy Aiken, „Feeding Germany: American Quakers in the Weimar Republic“, in: Diplomatic History 43, 4 (2019), 597-617; zu Russland David McFadden und Claire Gorfinkel, Constructive Spirit: Quakers in Revolutionary Russia (Pasadena: Intentional Productions, 2004). Zum Einsatz in Deutschland in den 1930er Jahren siehe Hans A. Schmitt, Quakers and Nazis: Inner Light in Outer Darkness (Columbia: University of Missouri Press, 1997). Zum Spanischen Bürgerkrieg Farah Mendlesohn, Quaker Relief Work in the Spanish Civil War (New York: Edwin Mellen Press, 2002).

18 Lynn Hunt, Inventing Human Rights: A History (New York/London: Norton, 2007); Richard Ashby Wilson und Richard D. Brown, „Introduction“, in: Richard Ashby Wilson und Richard D. Brown (Hg.), Humanitarianism and Suffering: The Mobilization of Empathy (Cambridge: Cambridge University Press, 2008), 1-28; Thomas Laqueur, „Bodies, Details, and the Humanitarian Narrative“, in: Lynn Hunt (Hg.), The New Cultural History (Berkeley/Los Angeles: University of California Press, 1989), 176-204; Thomas Laqueur, „Mourning, Pity, and the Work of Narrative in the Making of ,Humanity““, in: Richard Ashby Wilson und Richard D. Brown (Hg.), Humanitarianism and Suffering: The Mobilization of Empathy (Cambridge: Cambridge University Press, 2008), 31-57. 
Verhalten zeitige Folgen für Menschen außerhalb ihres unmittelbaren Lebensbereichs, desto mehr überquerte ihr Denken eine „humanitäre Schwelle“ und desto mehr begannen sie, sich für Menschen verantwortlich zu fühlen, denen sie nie begegnet waren und mit denen sie außer diesem Wissen nichts verband. ${ }^{19}$ Die Linie zur Entstehung eines quäkerspezifischen Humanitarismus zu ziehen, scheint insofern naheliegend, als nicht wenige unter den prominenten QuäkerAbolitionisten in England und den USA erfolgreiche Unternehmer waren. Tatsächlich haben viele Studien zur Anti-Sklaverei-Bewegung kritisch auf diesen Zusammenhang hingewiesen, indem sie den Quäker-Abolitionismus in einen Sinnzusammenhang mit der Anwendung ausbeuterischer Praktiken gegenüber „freien“ Arbeitern in den heimischen Fabriken oder auf den Kakaoplantagen und Zuckerrohrfeldern in Afrika und der Karibik stellten. ${ }^{20}$ Die These lässt sich fraglos nicht ohne Weiteres auf die humanitären Aktivitäten der Quäker im 20. Jahrhundert übertragen. Wie im Verlauf dieses Buches zu zeigen sein wird, findet sich jedoch ein Echo im Gegensatz zwischen der Bereitschaft des AFSC, notleidenden Kindern in Deutschland und der Sowjetunion, und dem zumindest anfänglichen Zögern, auch streikenden Bergarbeitern im eigenen Land $\mathrm{zu}$ helfen, das soziale und politische Widersprüche zu Tage treten ließ.

Vor diesem Hintergrund lassen sich die Hilfsaktivitäten der Quäker in den USA auch fruchtbar auf Forschungsansätze beziehen, die ausgehend von einigen klassischen anthropologischen und philosophischen Studien die soziale Funktion der „Gabe“ unterstreichen. ${ }^{21}$ Inwieweit die Quäker sich dabei ohne Weiteres in das gängige „sozialgeschichtliche Paradigma“ einfügen, das philanthropisches Handeln in erster Linie mit Blick auf die politischen Funktionen erklärt, die es aus Sicht gesellschaftlicher Eliten erfüllte, erscheint dabei als offene Frage. ${ }^{22}$ Während dieser Ansatz für das 19. Jahrhundert gut geeignet scheint, das wachsende hu-

19 Haskell, „Capitalism and the Origins of Humanitarian Sensibility: Part 1“; Thomas L. Haskell, „Capitalism and the Origins of Humanitarian Sensibility: Part 2“, in: American Historical Review 90, 3 (1985), 547-566.

20 Siehe z.B. David Brion Davis, „The Quaker Ethic and the Anti-Slavery International“, in: Thomas Bender (Hg.), The Anti-Slavery Debate: Capitalism and Abolitionism as a Problem in Historical Interpretation (Berkeley: University of California Press, 1992), 27-64; Jean Soderlund, Quakers and Slavery: A Divided Spirit (Princeton: Princeton University Press, 1988); James Walvin, The Quakers: Money and Morals (London: John Murray, 1997); Margaret Abruzzo, Polemical Pain: Slavery, Cruelty, and the Rise of Humanitarianism (Baltimore: John Hopkins University Press, 2011). 21 Siehe die klassischen Studien von Marcel Mauss, The Gift: Forms and Functions of Exchange in Archaic Societies (London/New York: Routledge, 2011); Jean Starobinski, Largesse (Chicago: The University of Chicago Press, 1997).

22 Alan J. Kidd, „Philanthropy and the ,Social History Paradigm““, in: Social History 21, 2 (1996), 180-192. 
manitäre Engagement vor allem von Gruppen am Rande der gesellschaftlichen Elite, namentlich von jüdischen Philanthropen, zu deuten, lassen sich die Quäker hier nicht ohne Weiteres einordnen. ${ }^{23}$ Während jüdische Philanthropen, so die These, auf doppelte Weise einen Statusgewinn erzielten - indem sie zum Teil der Hilfsgemeinschaft in den USA und zu Repräsentanten amerikanischer Hilfe im Ausland wurden - so war dies für die Quäker nur in bedingtem Maß relevant. Zwar behielten die Friends durch ihren Pazifismus stets eine gewisse Sonderstellung, die, wie die folgende Studie zeigen wird, der Quäkerhilfe im nationalen Kontext eine besondere Bedeutung verlieh. Andererseits besaß die Society of Friends Ende des 19. Jahrhunderts in den USA, anders als in England, keinen Außenseiterstatus im engeren Sinn. Vielmehr bildeten die Quäker hier insbesondere an der Ostküste einen anerkannten Teil des protestantischen Establishments. $^{24}$

Auch mit Blick auf genderspezifische Aspekte in der Entwicklung des humanitären Sektors, die Esther Möller, Katharina Stornig und Johannes Paulmann in einem jüngst erschienenen Sammelband hervorheben, bieten die Quäker ein interessantes Studienfeld. ${ }^{25}$ Nicht zuletzt ihr im Vergleich mit anderen protestantischen Denominationen hoher Status innerhalb der Religious Society of Friends, verlieh Quäkerinnen in der Geschichte der humanitären Hilfe eine herausragende Rolle. ${ }^{26}$ Gender bietet als „relational category that includes both femininity and masculinity “27 innerhalb der folgenden Studie im doppelten Sinn erweiterte Aufschlüsse: Mit Blick auf die Quäker wirft sie zum einen ein Schlaglicht auf die spezifische Rolle von Frauen und feministischen Netzwerken bei der Entwicklung des humanitären Sektors um den Ersten Weltkrieg. ${ }^{28}$ Zum anderen

23 Abigail Green, „Rethinking Sir Moses Montefiore: Religion, Nationhood, and International Philanthropy in the Nineteenth Century“, in: American Historical Review 110, 3 (2005), 631-658.

24 Thomas D. Hamm and Isaac Barnes May, „Conflict and Transformation, 1808-1920,“ in The Cambridge Companion to Quakerism, ed. Stephen W. Angell and Pink Dandelion (Cambridge: Cambridge University Press, 2018).

25 Esther Möller, Johannes Paulmann, and Katharina Stornig, (Hg.) Gendering Global Humanitarianism in the Twentieth Century: Practice, Politics and the Power of Representation (London: Palgrave Macmillan, 2020).

26 Elisabeth Potts Brown and Susan Mosher Stuard, Witnesses for Change: Quaker Women over Three Centuries (New Brunswick; London: Rutgers University Press, 1989).

27 Esther Möller, Johannes Paulmann, and Katharina Stornig, „Gendering 20th Century Humanitarianism: An Introduction,“ in: dies., Gendering Global Humanitarianism in the Twentieth Century: Practice, Politics and the Power of Representation, (London: Palgrave Macmillan, 2020), 3. 28 Mona L. Siegel, Peace on Our Terms : The Global Battle for Women's Rights after the First World War, Columbia Studies in International and Global History (New York City: Columbia University Press, 2020). 
rückt sie einen Prozess ins Bild, der als organisatorische „Vermännlichung“ der Quäkerhilfe beschrieben werden kann und der die Aufmerksamkeit für einen breiteren Trend zu schärfen vermag, in der humanitäre Hilfe als dezidiert „männliche“ Praxis re-interpretiert wurde.

\section{Humanitarismus und Religion}

Dass sich ein Buch zur Quäkerhilfe mit der Rolle der Religion als Triebfeder humanitärer Hilfe auseinandersetzt, liegt praktisch auf der Hand. Ein Verständnis von humanitärer Hilfe als einer im Kern religiösen Praxis - deren gemeinsamer Nenner die Berufung auf ein größeres und höheres Ganzes ist, das die Form Gottes, der Menschheit, der Idee sozialer Gerechtigkeit oder eben auch des Quäkerglaubens an „das von Gott in jedem Menschen“ annehmen kann - weist die Richtung, der dieses Buch folgen möchte. Auf eine einfache Formel gebracht, lässt sich sein Ansatz wie folgt beschreiben: Es möchte Religion als Antriebskraft humanitärer beziehungsweise sozialer Praxis ernst nehmen, ohne sie von ihrem historischen politischen, ökonomischen und gesellschaftlichen Kontext zu trennen. Es gilt dabei die Räume auszuloten, in denen zwischen individuellem Glauben und weltlichen Einflüssen der unterschiedlichsten Art Humanitarismus als eine Sozialform religiösen Handelns sichtbar wird. ${ }^{29}$ Wohlgemerkt: Wenn Glauben und Religion als Antriebsfedern humanitären Handelns in den Blick geraten, so geschieht dies in vollem Bewusstsein über die methodischen Grenzen des Anliegens. Historiker sollten im Unterschied zu Psychologen oder den Neurowissenschaften keinen Zugang zu den Köpfen ihrer Untersuchungsobjekte beanspruchen. Es geht vielmehr darum, den Motivationen im Diskurs zwischen Menschen nachzuspüren, im Prozess der Verständigung über Ziele und Sinngebung der Hilfe, wie er sich aus Berichten, Briefen, Sitzungsprotokollen und zuweilen aus Selbstzeugnissen erschließt.

Auch mit Blick auf die Verbindung zwischen Religion und Humanitarismus stützt sich die folgende Studie auf eine Fülle von Literatur zur faith-based aid auf nationaler wie auf internationaler Ebene. ${ }^{30}$ Sowohl für das 19. als auch das

29 Der Begriff der „Sozialformen religiösen Handelns“ lehnt sich an eine zentrale Kategorie der DFG-Forschergruppe „Transformation der Religion nach 1945“ an. Exemplarisch erscheint der Ansatz etwa in den Beiträgen in Wilhelm Damberg and Traugott Jähnichen (Hg.) Neue Soziale Bewegungen Als Herausforderung Sozialkirchlichen Handelns (Stuttgart: Kohlhammer, 2015).

30 Einen Überblick über die Bandbreite dieser Forschung liefern etwa die Beiträge in Michael Barnett and Janice Stein (Hg.) Sacred Aid: Faith and Humanitarianism (New York/Oxford: Oxford University Press, 2012). 
20. Jahrhundert liegt alleine für die USA eine Vielzahl von Studien vor - angefangen bei der Entstehung des social gospel bis zur Rolle der Kirchen beim Aufbau wohlfahrtsstaatlicher Einrichtungen in den USA während der Jahre des New Deal. ${ }^{31}$ Eine umfangreiche Literatur verbindet zudem die Entstehung des internationalen Humanitarismus mit dem gleichzeitigen Aufstieg des Evangelikalismus und der christlichen Missionsbewegung. Deren universeller Anspruch und globale Verbreitung im Zeitalter imperialer Ausdehnung bildeten eine Grundlage für das Entstehen persönlicher und ideeller Netzwerke, die als Kanäle für humanitäre Aktivitäten bereitstanden. ${ }^{32}$ Insbesondere für die USA haben Studien wiederholt den Einfluss evangelikal geprägter moral reformers und Missionare bei der Entstehung eines global ausgerichteten Humanitarismus betont. ${ }^{33}$ Dabei fügen sich die Aktivitäten der Quäker in diesem Kontext selten leicht ins allgemeine Bild ein: Wenngleich sie in den USA und Großbritannien eigene Missionsgesellschaften gründeten, wurde die Missionsbewegung im Gegensatz zu den meisten anderen protestantischen Denominationen zu keinem Zeitpunkt ein vollständig akzeptierter Teil der Society of Friends als Ganzer. Gleiches galt für den Evangelikalismus, der Teile der Quäker erfasste, dem es jedoch nie gelang, eine hegemoniale Stellung innerhalb der Gesellschaft $\mathrm{zu}$ erlangen. ${ }^{34}$ Auf diese Weise spielten Quäkermissionare und ein evangelikaler Impuls im Rahmen einzelner Hilfseinsätze durchaus eine Rolle. Gleichzeitig, wie insbesondere für den Untersuchungszeitraum zu zeigen sein wird, geschah dies stets in einem Spannungs-

31 Michael G. Thompson, For God and Globe: Christian Internationalism in the United States between the Great War and the Cold War (Ithaca: Cornell University Press, 2015).

32 Ian Tyrell, Reforming the World: The Creation of America's Moral Empire (Princeton: Princeton University Press, 2010); Amanda Porterfield, „Protestant Missionaries: Pioneers of American Philanthropy“, in: Lawrence J. Friedman und Mark D. McGarvie (Hg.), Charity, Philanthropy, and Civility in American History (Cambridge: Cambridge University Press, 2003), 49-71.

33 David A. Hollinger, Protestants Abroad : How Missionaries Tried to Change the World but Changed America (Princeton, New Jersey: Princeton University Press, 2017). Ein evangelikaler Impuls war etwa bei der Gründung der Rotkreuzbewegung maßgeblich: John F. Hutchinson, „Rethinking the Origins of the Red Cross“, in: Bulletin for the History of Medicine 63, 4 (1989), 557578. In einem breiteren Kontext: Bertrand Taithe, „Horror, Abjection and Compassion: From Dunant to Compassion Fatigue“, in: New Formations 62 (2007), 123-136. Zur Heilsarmee siehe Harald Fischer-Tiné, „Global Civil Society and the Forces of Empire: The Salvation Army, British Imperialism and the ,Pre-History“ of NGOs (Ca. 1880-1920)“, in: Sebastian Conrad und Dominic Sachsenmaier (Hg.), Competing Visions of World Order: Global Moments and Movements, 1880s 1930s (New York: Palgrave Macmillan, 2007), 29-67.

34 Zur allgemeinen Einordnung siehe Matthew Avery Sutton, American Apocalypse : A History of Modern Evangelicalism (Cambridge, Massachusetts: Belknap Press of Harvard University Press, 2014); Elesha J. Coffman, The Christian Century and the Rise of the Protestant Mainline (New York: Oxford University Press, 2013). 
verhältnis zu jenen Quäkern, die sich teils entschieden gegen jede Art missionarischer Tätigkeit und folglich auch die Verquickung von message und relief work stellten. ${ }^{35}$

Hier gilt es im Folgenden einzelne Aspekte der theologischen Grundsätze und sozialen Praxis des Quäkertums in Beziehung mit dem „Markenkern“ humanitärer Hilfe zu setzen, der sowohl für das Selbstbild als auch die Außendarstellung des AFSC prägend wurde. Ein besonderes Augenmerk gilt dabei dem als „Friedenszeugnis“ (peace testimony) bekannten, religiös begründeten Pazifismus der Quäker, der in allen Hilfseinsätzen eine mehr oder weniger herausragende Rolle spielte. ${ }^{36}$ In gleicher Weise fügt sich die Geschichte des Quäkerhumanitarismus auf ganz spezifische Weise in die breitere Entwicklung einer „social christianity“ seit dem 19. Jahrhundert ein. Die Einbindung einzelner Quäkerinnen und Quäker sowie des AFSC als Resonanzboden und Impulsgeber der verschiedensten sozialen Reformanliegen als Teil einer breiteren Bewegung der protestantischen Kirchen in den USA sowie der Austausch mit säkularen Gruppen stellt einen wichtigen Bezugspunkt der Arbeit dar. ${ }^{37}$

Wie auch immer jedoch einzelne Glaubenselemente, theologische Paradigmenwechsel und das Engagement für weltliche Anliegen sich auf die humanitären Aktionen der Quäker auswirkten, eine Verbindung blieb stets erhalten. Ungeachtet aller Wandlungen und Konvergenzen mit dem entstehenden humanitären „Sektor“, zu dessen Teil das AFSC im Beobachtungszeitraum wurde: Es blieb stets eine im Quäkertum verwurzelte Organisation und verstand sich als Vertreter der Society of Friends. Die Repräsentation seines Handelns und deren Antriebskräfte - nach außen wie nach innen - waren und blieben in aller Regel von religiösen Motiven geprägt. Wie Guy Aiken zu Recht feststellt, bezeichnete sich das AFSC in den 1920er und 1930er Jahren selbst äußerst selten als eine humanitäre Agentur im engeren Sinne, „but instead thought of itself as an agency for the Christianization of the international and social order - that is as a vehicle

35 Greenwood, Quaker Encounters Vol. 1: Friends and Relief, 1-18; John Ormerod Greenwood, Quaker Encounters Vol. 2: Vines on the Mountains (York: William Sessions Limited, 1977); John Ormerod Greenwood, Quaker Encounters Vol. 3: Whispers of Truth (York: William Sessions Limited, 1979); Tusan, „The Business of Relief Work: A Victorian Quaker in Constantinople and Her Circle“.

36 Elaine Bishop und Jiseok Jung, „Seeking Peace: Quakers Respond to War“, in: Stephen W. Angell und Pink Dandelion (Hg.), The Cambridge Companion to Quakerism (Cambridge: Cambridge University Press, 2018), 106-127.

37 Siehe zum Beispiel zur Verknüpfung mit der politischen Linken in den USA Vaneesa Marie Cook, Spiritual Socialists: Religion and the American Left (Philadelphia: University of Pennsylvania Press, 2019). 
of social Christianity and the Social Gospel““.38 Ein besonders hoher Wert gerade mit Blick auf den oben skizzierten allgemeinen Ansatz, der individuelle und kollektive Zugänge verbindet, kommt den Arbeiten der Anthropologin Ilana Feldman zu. Ihre Studien anhand eines späteren Einsatzes des AFSC - unter palästinensischen Flüchtlingen nach dem Zweiten Weltkrieg - arbeiten exemplarisch die Spezifika des Quaker Way der Hilfe heraus, der „ethical labor“ und „humanitarian relief“ miteinander verbindet. ${ }^{39}$

\section{Quaker relief und American Empire}

Zuletzt bezieht diese Studie wichtige Inspiration auch aus Forschungsbeiträgen, welche die Anfänge grenzüberschreitender humanitärer Hilfe in den Kontext imperialer Politik und Ausdehnung seit Ende des 19. Jahrhunderts stellen. ${ }^{40} \mathrm{Hu}-$ manitäre Hilfe findet sich hier verknüpft sowohl mit politisch und religiös aufgeladenen Ideen der civilizing mission, die als solche den verschiedenen imperialen Projekten Legitimität verliehen. ${ }^{41}$ Für den vorliegenden Fall besonders fruchtbar sind Studien zu den USA, die die Rolle privater Akteure, von Unternehmern, Missionaren oder Philanthropen im Kontext des amerikanischen Aufstiegs zur Weltmacht betonen. Die Hilfsaktionen privater Gruppen und Komitees seit dem letzten Drittel des 19. Jahrhunderts erscheinen hier oftmals als das wohlwollende Gesicht amerikanischen Hegemoniestrebens, dessen Hauptfunktion darin bestand, harten diplomatischen und wirtschaftlichen Interessen im Ausland Türen zu öffnen. Gleichzeitig schreibt diese Literatur der humanitären Hilfe eine bedeutende Rolle zu, wenn es galt in den USA selbst in der öffentlichen Debatte den Boden für eine interventionistische Außenpolitik zu bereiten. In diesem Sinn erscheinen humanitäre Hilfsorganisationen in manchen klassischen Werken als „chosen instrument“ der US-amerikanischen Außenpolitik und als integraler Bestandteil früher cultural diplomacy. ${ }^{42}$

38 Guy Aiken, „Social Christianity and the American Friends Service Committee’s Pacifist Humanitarianism in Germany and Appalachia, 1919-1941“ (Ph.D., University of Virginia, 2017), 3. 39 Ilana Feldman, „The Quaker Way: Ethical Labor and Humanitarian Relief,“ American Ethnologist 34, 4 (2007).

40 Baughan und Everill, Special Issue: Empire and Humanitarianism.

41 Boris Barth und Jürgen Osterhammel (Hg.), Zivilisierungsmissionen: Imperiale Weltverbesserung seit dem 18. Jahrhundert (Konstanz: UKV Verlag, 2005).

42 Tyrell, Reforming the World: The Creation of America's Moral Empire; Emily S. Rosenberg, Spreading the American Dream: American Cultural and Economic Expansion, 1890-1945 (New York: Hill and Wang, 1982). Einige Beiträge in Brendan Simms und D. J. B. Drim (Hg.), Humani- 
Erneut fällt es schwer, die Quäker in diese Erzählung einzuordnen, vor allem im Hinblick auf die dezidiert anti-imperialistischen und anti-interventionistischen Positionen, die viele Friends vor dem Hintergrund der Diskussionen um die Rolle der USA im „großen imperialistischen Spiel“ des späten 19. und beginnenden 20. Jahrhunderts einnahmen. Im Gegensatz zu anderen humanitären Akteuren, namentlich dem Amerikanischen Roten Kreuz, hielt die Society of Friends aus einer Reihe von, in der religiösen und sozialen Tradition der Quäker wurzelnden, Gründen lange Zeit Distanz zu staatlichen Stellen.

Wie dieses Buch zeigen wird, brachte erst der Erste Weltkrieg einen Wandel. Erst dann, und im Wesentlichen sogar erst nach dem Eintritt der USA in den Ersten Weltkrieg, wurden die Quäker zum quasi-offiziellen und erkennbaren Teil eines US-amerikanischen humanitären „Komplexes“. Doch selbst dann blieb ihrer Teilnahme ein Moment der Ambivalenz erhalten. Meine These in dieser Studie lautet, dass die Quäker, indem sie zu einem der vielen Gesichter des amerikanischen Humanitarismus im Ausland wurden, auf doppelte Weise am Prozess der globalen Expansion der USA teilnahmen: als Agenten, die American values, amerikanische Technik und amerikanische Macht nach außen projizierten einerseits; andererseits indem sie zu Hause am Definitionskampf um die Frage teilnahmen, was die amerikanische Nation und ihre Außenpolitik in ihrem Wesen von anderen Nationen unterschied oder unterscheiden sollte.

Damit ist auch ein methodischer Ansatz angesprochen, der für die folgende Arbeit bestimmend ist. Die Eigenarten, welche die humanitäre Identität der amerikanischen Quäker auszeichnen, werden dabei in Beziehung zu deren Einbettung in eine Matrix aus transnationalen Verbindungen sowie einen spezifisch nationalen Kontext untersucht. Unter diesem Gesichtspunkt ordnen sich die Hilfseinsätze des AFSC in der Zwischenkriegszeit in einer breiteren Sicht in Forschungsansätze ein, die sich mit der „transnational USA“ befassen. Anschließend an Studien, welche die USA nicht als Nation sui generis, sondern vielmehr als „Nation among Nations“ verstehen, deren Eigenheiten im ständigen Dialog mit der Welt außerhalb der USA entstanden, wird in der folgenden Studie auch die Hilfstätigkeit der amerikanischen Quäker als Ergebnis eines Austauschverhältnisses beschrieben. Die humanitären Aktivitäten des AFSC erscheinen dabei als eines der vielfältigen „Atlantic Crossings“ der Zeit, bei dem die Quäker als Ver-

tarian Intervention: A History (Cambridge: Cambridge University Press, 2011) weisen in die gleiche Richtung. 
treter eines spezifisch US-amerikanischen brand der Hilfe wahrgenommen wurden. ${ }^{43}$

Der Austausch mit einer Vielzahl von Akteuren in und außerhalb der USA, der resultierende Prozess permanenter Selbstbefragung hinsichtlich der eigenen Position, der Motive und Ziele der Hilfe, stehen im Mittelpunkt der Studie. Das Feld der Akteure reicht dabei von deutschen und sowjetischen Regierungsbehörden bis ins Weiße Haus, von den politischen Sympathisanten der Spanischen Republik bis zu streikenden Bergarbeitern in West-Virginia und von evangelikalen Missionaren bis zu jüdischen Flüchtlingsorganisationen. Die Reputation, welche die Quäkerhilfe im Untersuchungszeitraum erlangte, ihr unverwechselbarer „Markenkern“ erscheinen in diesem Licht weniger als Ergebnis einer langen Tradition, als vielmehr als Resultat eines kontingenten, stetig fortlaufenden Aushandlungsprozesses, in dem jede Aktion auf nationaler wie auf internationaler Ebene neue Impulse erzeugte, die ihrerseits auf die Aktionen im In- und Ausland rückwirkten.

\section{Quäkerhilfe im Zeitalter des humanitären Aufbruchs}

Die Gründung des AFSC und seine Hilfsaktionen während der Jahre zwischen den beiden Weltkriegen soll im Folgenden vor dem Hintergrund der breiteren Entwicklungen des humanitären Sektors in diesem Zeitraum beschrieben werden. Private Hilfsorganisationen wie das AFSC erscheinen innerhalb dieser Geschichte als ein zentraler Teil eines dichter werdenden transnationalen Netzes der Hilfe, das private, staatliche und überstaatliche Institutionen umfasste. In den Blick gerückt wird damit die Bedeutung der Zwischenkriegszeit als formative Phase in der Entwicklung eines modernen humanitären „Regimes“. ${ }^{44}$ Kein Zweifel: Nicht alles war neu in dieser Phase. Die Geschichte des Internationalen Roten Kreuzes begann ebenso wenig mit dem Ersten Weltkrieg wie die humanitären Aktivitäten

43 Thomas Bender, A Nation among Nations: America's Place in World History (New York: Hill and Wang, 2006); Daniel T. Rodgers, Atlantic Crossings: Social Politics in a Progressive Age (Cambridge, MA: Harvard University Press, 1998).

44 Daniel Gorman, The Emergence of International Society in the 1920s (Cambridge: Cambridge University Press, 2012); Daniel Laqua (Hg.), Internationalism Reconfigured: Transnational Ideas and Movements between the World Wars, International Library of Twentieth Century History (London/New York: I.B. Tauris, 2011). Mit Fokus auf den Völkerbund siehe Glenda Sluga, Internationalism in the Age of Nationalism (Philadelphia: University of Pennsylvania Press, 2013), 45-75; Sönke Kunkel und Christoph Meyer (Hg.), Aufbruch ins postkoloniale Zeitalter: Globalisierung und die aussereuropäische Welt in den 1920er und 1930er Jahren (Frankfurt am Main: Campus, 2012). 
US-amerikanischer Hilfskomitees und -vereinigungen für die Opfer von Krieg, Verfolgung und Hungersnöten..$^{45}$ Die „humanitären Interventionen“ europäischer Mächte zugunsten der Armenier und anderer verfolgter christlicher Minderheiten im Osmanischen Reich hierfür nur ein Beispiel für Kontinuitätslinien, die bis weit ins 19. Jahrhundert zurückreichen. ${ }^{46}$ Michael Barnetts Ansatz, die gesamte Periode von 1800 bis 1945 als Phase des ,,imperial humanitarianism“ zu konzipieren, die erst nach dem Zweiten Weltkrieg von einem „Neo-humanitarianism“ abgelöst worden sei, erscheint dagegen zu grobkörnig, ${ }^{47}$ um die tiefen Brüche einzufangen, die sich angestoßen durch den Krieg und die nachfolgende Friedensordnung auftaten. Mein Argument lautet, dass die „Zwischenkriegszeit“ vor allem als die Phase in der Geschichte der humanitären Hilfe beschrieben werden kann, in der diese Hilfe nicht nur auf mehreren Ebenen, der nationalen, internationalen und transnationalen, massiv expandierte, sondern in der diese Dimensionen auch zunehmend miteinander korrespondierten. Eine zweite Besonderheit besteht in dem enormen Bedeutungsgewinn US-amerikanischer Hilfe und daraus abgeleitet deren überwiegend transatlantischen Ausrichtung während dieser Phase. Davon ausgehend erscheint die Zeit zwischen den beiden Weltkriegen eingebettet in die breitere Periodisierung, die Norbert Götz, Georgina Brewis und Steffen Werther in ihrer jüngsten Studie zur „moral economy of famine relief“ entlang sich verändernder politisch-ökonomischer „Regimes“ vornehmen. Aus diesem Blickwinkel beschreibt der Wechsel vom 19. zum 20. Jahrhundert den Übergang von einem „ad hoc humanitarianism“ spontaner, anlässlich konkreter Hilfsanlässe gebildeter Komitees zum „organised humanitarianism“ des neuen Zeitalters, der in den Grundzügen dem Aufkommen von „Taylorism and mass society“ entsprach. Die folgende Studie schließt sich dieser Sichtweise an und modifiziert sie durch das

45 Dieter Riesenberger, Für Humanität in Krieg und Frieden: Das Internationale Rote Kreuz $1863-$ 1977 (Göttingen: Vandenhoeck \& Ruprecht, 1992); Merle Curti, American Philanthropy Abroad (New Brunswick, NJ: Rutgers University Press, 1963).

46 Davide Rodogno, Against Massacre: Humanitarian Interventions in the Ottoman Empire, 18151914. The Emergence of a European Concept and International Practice (Princeton: Princeton University Press, 2012); Fabian Klose (Hg.), The Emergence of Humanitarian Intervention: Ideas and Practice from the Nineteenth Century to the Present (Cambridge: Cambridge University Press, 2015).

47 Barnett, Empire of Humanity: A History of Humanitarianism. Daneben bieten einen guten Überblick Paulmann, „Conjunctures in the History of Humanitarian Aid During the Twentieth Century“; Taithe, „Horror, Abjection and Compassion: From Dunant to Compassion Fatigue“. Eine Zusammenfassung und Synthese der jüngeren Forschung bietet Eleanor Davey, A History of the Humanitarian System: Western Origins and Foundations, HPG Working Papers (June 2013). 
stärkere Gewicht, das sie dem Ersten Weltkrieg als Katalysator des Übergangs beimisst. $^{48}$

Die Bruchlinien, in die sich die Geschichte des Quäkerhumanitarismus während der Zwischenkriegszeit einordnet, werden dabei vor allem auf drei Feldern identifiziert: Es geht erstens um die beispiellose Mobilisierung humanitärer Hilfe im Zeichen ,,totaler“ Kriegführung während des Ersten Weltkriegs. Darüber hinaus rückt zweitens der Aufstieg der USA zur „humanitären Supermacht“ in ihrem Verhältnis zum Bedeutungsgewinn und zur „Professionalisierung“ privater Hilfsorganisationen ins Bild. Drittens gerät das Erscheinen neuer Akteure, in der Gestalt internationaler Organisationen wie des Roten Kreuzes und des Genfer Völkerbundes, als eine neue Entwicklung in den Blick.

Am Anfang steht der Erste Weltkrieg als Epochenbruch mit weitreichenden Folgen auch für die Geschichte der Hilfe. ${ }^{49}$ Das schiere Ausmaß des Leidens von Soldaten und Zivilisten in einer Auseinandersetzung, die zunehmend den Charakter eines „totalen Krieges“ annahm, schuf Voraussetzungen, die humanitären Aktivitäten ein gänzlich neues Gewicht verliehen. ${ }^{50}$ In der Arbeit für die Opfer auf den Schlachtfeldern sowie für das unüberschaubare Heer der Kriegsgefangenen - ca. neun Millionen Soldaten gerieten von 1914 bis 1918 in Gefangenschaft - erlebte etwa das 1863 gegründete Internationale Rote Kreuz eine Art zweite Geburtsstunde. ${ }^{51}$ Auch andere Organisationen wie die angloamerikanische Young Men's Christian Association (YMCA) wurden auf diesem Feld aktiv und erhielten so die Möglichkeit, ihre Reichweite auszudehnen. ${ }^{52}$ Auf nationaler Ebene erfüllte die Arbeit für die Kriegsgefangenen darüber hinaus eine wichtige Funktion bei der Mobilisierung der „Heimatfront“, indem sie eine Brücke zwischen Soldaten und Zivilisten schlug. Nirgendwo wurde dieser Zusammenhang deutlicher als in den USA, nachdem diese unter Präsident Woodrow Wilson im April 1917 an der Seite der Entente in den Krieg eingetreten waren. Insbesondere das Amerikanische Rote Kreuz tat sich dabei hervor, die Hilfe für die eigenen Soldaten sowie für die Zi-

48 Götz, Brewis und Werther, Humanitarianism in the Modern World: The Moral Economy of Famine Relief, 4.

49 Bruno Cabanes, The Great War and the Origins of Humanitarianism, 1918-1924 (Cambridge: Cambridge University Press, 2014).

50 Aus der wachsenden Fülle der Literatur sei exemplarisch hingewiesen auf die Spezialausgabe Humanitarianism in the First World War, First World War Studies 5, 1 (2014).

51 Uta Hinz, „Humanität im Krieg? Internationales Rotes Kreuz und Kriegsgefangenenhilfe Im Ersten Weltkrieg“, in: Jochen Oltmer (Hg.), Kriegsgefangene im Europa des Ersten Weltkriegs. (Paderborn: Schöningh, 2006), 216-236: hier 221.

52 Tammy Proctor, Civilians in a World at War, 1914-1918 (New York: New York University Press, 2010), 153-203, hier 185-187. 
vilbevölkerung in den verbündeten Ländern als „patriotic obligation“ und mithin als Teil der nationalen Identität zu propagieren. ${ }^{53}$ Eine Vielzahl weiterer christlicher, jüdischer und ethnisch definierter Hilfskomitees ordnete sich in diese Bewegung ein. ${ }^{54}$ Stärker noch als in anderen Ländern übernahmen amerikanische Hilfsorganisationen wie das ARC oder das von Herbert Hoover geleitete Committee on the Relief of Belgium (CRB) ${ }^{55}$ auch erstmals eine quasi-diplomatische Rolle. Sie wurden umgekehrt in Europa als Repräsentanten nicht nur amerikanischer Großzügigkeit und Projektion des wachsenden weltpolitischen Gewichts der USA, sondern auch eines spezifisch US-amerikanischen, von wissenschaftlichen Prinzipien geleiteten humanitären „Stils“ wahrgenommen. ${ }^{56}$

Der Erste Weltkrieg zeitigte auch noch in einer anderen, teils gegenläufigen Hinsicht Folgen: Gerade jenen, die sich der Mobilisierung für den Krieg etwa aus pazifistischer Überzeugung verweigerten, bot ein humanitäres Engagement eine der wenigen verfügbaren Nischen. Dies galt für jene, darunter viele Quäker, für die ein Dienst in Sanitäts- und Wiederaufbaueinheiten hinter der Front eine Alternative zum Dienst an der Waffe darstellte. Dies galt umso mehr für jene an der Heimatfront, die sich für Zivilinternierte engagierten, das heißt Staatsangehörige feindlicher Nationen, die sich bei Kriegsbeginn auf dem Boden der jeweils „falschen" Seite befanden und den Krieg in der Regel in Internierungslagern verbrachten. ${ }^{57}$ Gerade diese Arbeit für die enemy aliens, wie sie in englischsprachigen Ländern bezeichnet wurden, schuf Foren für die Friedensarbeit in Kriegszeiten und war einer der wenigen offenen Kanäle für die Zusammenarbeit über Frontlinien hinweg. Wie zu zeigen sein wird, gingen einige der einflussreichen huma-

53 Julia Irwin, Making the World Safe: The American Red Cross and a Nation's Humanitarian Awakening (New York: Oxford University Press, 2013). Bereits vor dem Ersten Weltkrieg hatte sich die Arbeit des ARC von seinen Schwestergesellschaften in anderen Ländern dadurch unterschieden, das es sich neben der reinen Versorgung von Kriegsopfern bereits auch Hilfseinsätzen in Friedenszeiten widmete. Dieses ausgedehnte Mandat ging als „American Amendment“ unmittelbar nach dem Ende des Ersten Weltkriegs in die Satzung des Internationalen Roten Kreuzes ein. Siehe auch Riesenberger, Für Humanität in Krieg und Frieden: Das Internationale Rote Kreuz 1863-1977, 86-89.

54 Granick, „Waging Relief. The Politics and Logistics of American Jewish War Relief in Europe and the near East (1914-1918)“.

55 Zur CRB vor allem George H. Nash, The Life of Herbert Hoover: The Humanitarian, 1914-1917 (New York: Norton, 1983).

56 Irwin, Making the World Safe: The American Red Cross and a Nation's Humanitarian Awakening.

57 Matthew Stibbe, Civilian Internment During the First World War: A European and Global History, 1914-1920 (London: Palgrave Macmillan, 2019). 
nitären Organisationen der Nachkriegszeit, wie das britische Kinderhilfswerk Save the Children, aus dieser Zusammenarbeit hervor. ${ }^{58}$

Dies war freilich nur eine der Konsequenzen des Krieges, die über das Ende der Feindseligkeiten hinauswirkten. Die Umwälzungen, die ihm folgten, mithin die Friedensordnung von Versailles selbst, erzeugten neuen Handlungsbedarf. Die Flüchtlingsbewegungen, die sich als Folge des Zusammenbruchs der alten Ordnung und der in Versailles neu gezeichneten Landkarte in Gang setzten revolutionäre Umwälzungen und Bürgerkriege schufen eine Fülle neuer humanitärer Krisen. Eines der auffälligen Resultate des Krieges war, dass sich nun viele Augen auf die USA richteten, wenn es galt, durch die Versorgung der Notleidenden zur Stabilisierung der sozialen und politischen Lage in Europa und darüber hinaus beizutragen..$^{59}$

Die neuere Forschung hat darüber hinaus ein Schlaglicht auf die immens gewachsene Bedeutung nichtstaatlicher Hilfsorganisationen und deren zunehmende „Professionalisierung“ geworfen. Anders als die in der Regel ad hoc und anlässlich konkreter Krisen gegründeten Komitees der Vorkriegsjahrzehnte, arbeiteten viele der neu entstandenen NGOs mit einem festen Stab von Mitarbeitern und beschäftigten Experten für ein differenziertes Feld von Aufgabenbereichen von der Öffentlichkeitsarbeit bis zu logistischen Fragen der Hilfseinsätze, und inkorporierten medizinisches, ernährungswissenschaftliches und anderes relevantes Wissen. Humanitäre Hilfe wurde erstmals zur Karriereoption.

Eine, wenngleich keinesfalls exklusive, Führungsrolle nahmen abermals die USA ein. Das ARC sowie die halbstaatliche American Relief Administration (ARA) ${ }^{60}$ des späteren US-Präsidenten Herbert Hoover verkörperten einen „American Way“ humanitärer Hilfe, der von einem wissenschaftlichen Impetus, von Ideen von Selbsthilfe und langfristigem Wiederaufbau getragen wurde. Diesen beiden halb-

$58 \mathrm{Zu}$ Save the Children siehe vor allem: Emily Baughan, Saving the Children: Humanitarianism, Internationalism and Empire, 1915-1970 (Berkeley: University of California Press, 2021). Emily Baughan, „Every Citizen of Empire Implored to Save the Children! Empire, Internationalism and the Save the Children Fund in Inter-War Britain“, in: Historical Research 86, 231 (2013), 116-137; Rodney Breen, „Saving Enemy Children: Save the Children's Russian Relief Operation, 19211923“, in: Disasters 18, 3 (1994), 221-238; Baughan, Saving the Children: Humanitarianism, Internationalism and Empire, 1915-1970.

59 Maul, „The Rise of a Humanitarian Superpower: American NGOs and International Relief, 1917-1945.“

60 Zur ARA als Organisation liegen keine Monografien vor. Einen Überblick aus unterschiedlichen Blickwinkeln bieten Bertrand M. Patenaude, The Big Show in Bololand: The American Relief Expedition to Soviet Russia in the Famine of 1921 (Stanford: Stanford University Press, 2002); Curti, American Philanthropy Abroad; Leo Eugene Chavez, Herbert Hoover and Food Relief: An Application of American Ideology (Univ.-Diss., Ann Arbor: University of Michigan, 1976). 
oder parastaatlichen Institutionen schlossen sich eine ganze Reihe von Nichtregierungsorganisationen an, die im Windschatten von ARC und ARA und oftmals mit deren finanzieller und logistischer Unterstützung ihre Aktivitäten ausbauten: $\mathrm{Zu}$ diesen Organisationen gehörte etwa das 1914 gegründete American Jewish Joint Distribution Committee, ${ }^{61}$ dessen Aufmerksamkeit insbesondere der jüdischen Bevölkerung in Russland sowie in den Ländern Ost- und Südosteuropas galt. Dazu gehörten auch der evangelikal geprägte Near East Relief, ${ }^{62}$ der vor allem zugunsten der Überlebenden des armenischen Genozids von 1915 aktiv wurde, sowie eine ganze Reihe weiterer religiöser und säkularer Gruppen.

Ein Teil der Forschung betont vor diesem Hintergrund seit langem die Bedeutung humanitärer NGOs als Agenten des „,American Empire““.63 Inwieweit sich dieses Label auf die Arbeit des American Friends Service Committee anwenden lässt, ist eine der Grundfragen der folgenden Arbeit. Inspiration bezieht sie dabei nicht nur aus der Forschung zu den USA, sondern auch zum British Empire. Im Falle der Quäker, deren humanitäre Unternehmungen in aller Regel eine transnationale beziehungsweise angloamerikanische Dimension aufwiesen, erscheinen auch die Erfahrungen von Organisationen wie Save the Children einschlägig, denen die jüngere Forschung eine bedeutende Rolle dabei zuschreibt, das Bild des British Empire als einer moralischen, zivilisatorischen Kraft gefestigt, und damit eine Art des ,,humanitarian Imperialism“ befördert zu haben. ${ }^{64}$

Das Verhältnis dieser neuartigen Form humanitärer Organisationen zu ihren jeweiligen Herkunftsstaaten ist ein zentrales Thema dieses Buches. Dabei werden dichotomische Sichtweisen verworfen, die NGOs entweder als bloße Agenten staatlicher Politik oder in romantisierender Sicht als im Kern politikferne und einem rein humanitären Ethos verpflichtete Akteure betrachten. Es schließt vielmehr an die neuere Forschung zum „Internationalismus im Zeitalter des Nationalismus“ an, die die Verknüpfungen zwischen staatlichem und „privatem“ Handeln betont, und wendet dieses Internationalismus-Konzept auf die bislang noch kaum beachtete Geschichte der humanitären Hilfe als Teil der Geschichte

$61 \mathrm{Zu}$ den Anfängen des JDC siehe Jaclyn Granick, International Jewish Humanitarianism in the Age of the Great War (Cambridge: Cambridge University Press, 2021).

62 Keith David Watenpaugh, Bread from Stones: The Middle East and the Making of Modern Humanitarianism (Oakland: University of California Press, 2015).

63 Emily S. Rosenberg, „Missions to the World: Philanthropy Abroad,“ in Charity, Philanthropy, and Civility in American History, Lawrence J. Friedman and Mark D. McGarvie (Hg.) (Cambridge: Cambridge University Press, 2003). 241-258.

64 Aus der zahlreichen Literatur, die die Funktion von NGOs hervorhebt, siehe etwa Amalia Ribi Forclaz, Humanitarian Imperialism: The Politics of Anti-Slavery Activism, 1880-1940 (Oxford: Oxford University Press, 2015); Baughan, „Every Citizen of Empire Implored to Save the Children! Empire, Internationalism and the Save the Children Fund in Inter-War Britain“. 
internationaler Praxis an. ${ }^{65}$ Dadurch fällt, durch die Linse der Quäker, auch nicht zuletzt ein Licht auf die während der Zwischenkriegszeit gewachsene Bedeutung internationaler Institutionen für die humanitäre Hilfe. Insbesondere der 1919 gegründete Genfer Völkerbund übernahm hier eine bedeutende Rolle, die allerdings lange Zeit im Schatten der Frage nach dem „Scheitern“ der friedenspolitischen Funktionen des Völkerbundes wenig Beachtung gefunden hat. Mittlerweile hat sich manches geändert: Eine Vielzahl von Studien ist seit 2007 Susan Pedersens Aufruf gefolgt, zum Völkerbund „zurückzukehren“, und beleuchtet seither aus unterschiedlichen Perspektiven den Einfluss, den die „technischen“ Aktivitäten dieses ersten „experiment in world government“ in einer Vielzahl von Bereichen, von der Gesundheitspolitik bis zur Weltwirtschaft, ausübten. ${ }^{66}$

In der folgenden Arbeit rückt der Völkerbund - neben anderen Institutionen wie dem Internationalen Roten Kreuz (dem älteren ICRC und der 1919 gegründeten International League of Red Cross Societies ${ }^{67}$ ) - vor allem in seiner Funktion als Anlaufstelle und Kristallisationspunkt für humanitäre NGOs in den Mittelpunkt. ${ }^{68}$ Aus dem Blickwinkel des AFSC gerät dabei insbesondere der Bereich der Flüchtlingshilfe in den Fokus, der als Gradmesser für die Möglichkeiten und gleichzeitig für die Grenzen internationalen humanitären Handelns in der Zwischenkriegszeit dienen wird. Wenngleich der Anspruch des Völkerbundes, der ILO und anderer seiner Untergliederungen im Bereich der Flüchtlingspolitik als Koordinatorin privater und staatlicher Aktivitäten $\mathrm{zu}$ dienen, sich selten im gewünschten Maße realisieren ließ: ${ }^{69}$ Die folgende Arbeit kann zeigen, wie gerade in

65 Glenda Sluga, Internationalism in the Age of Nationalism (Philadelphia: University of Pennsylvania Press, 2013).

66 Susan Pedersen, „Back to the League of Nations“, in: American Historical Review 112, 4 (2007). 67 Riesenberger, Für Humanität in Krieg und Frieden: Das Internationale Rote Kreuz 1863-1977. Zum IKRK siehe David P. Forsythe, The Humanitarians: The International Committee of the Red Cross (Cambridge: Cambridge University Press, 2005). Zu den Anfängen des Roten Kreuzes Hutchinson, „Rethinking the Origins of the Red Cross“.

681924 wurde zu diesem Zweck eine ständige Konferenz privater Hilfsorganisationen beim Hochkommissariat für die Flüchtlinge eingerichtet. Umgekehrt entsandten die Vertreter der wichtigsten Organisationen von Save the Children über die Quäker bis hin zu jüdischen Hilfskomitees ihre Vertreter in einen Ausschuss, der den Hochkommissar in seiner Arbeit beriet - eines der umfassendsten und letztlich gescheiterten, in jedem Fall aber vergessenen Projekte zur Einführung einer Art gegenseitigen humanitären Krisenversicherung: John F. Hutchinson, „Disasters and the International Order: Earthquakes, Humanitarians, and the Ciraolo Project“, in: International History Review 22, 1 (2000), 1-36.

$69 \mathrm{Zu}$ den Völkerbundaktivitäten auf dem Feld der Flüchtlingspolitik siehe Claudena Skran, Refugees in Interwar Europe: The Emergence of a Regime (Oxford: Clarendon Press, 1995). 
diesem Bereich erste Mechanismen potenziell globaler humanitärer governance entstanden. Sie wirft dabei ebenfalls ein Licht auf die durchaus proaktive Rolle, die transnational agierende humanitäre NGOs wie das AFSC in diesem Kontext übernahmen, wenn sie ihrerseits als Broker zwischen staatlichen und internationalen Institutionen auftraten. Der besondere Reiz, sich den Quäkern von dieser Seite zu nähern, liegt gerade in ihrer ambivalenten Haltung gegenüber Internationalismus als Ideologie und Praxis. Internationales Handeln wurde von den Quäkern des AFSC, wie zu zeigen sein wird, in zunehmendem Maß als Teil der humanitären Identität angesehen. Der Wert der ihm beigemessen wurde, die Ausdrucksformen, die das AFSC ihm gab, und nicht zuletzt die „Interpretation“ (um Quäkerterminologie zu verwenden) für die eigene Klientel war stets mit Überlegungen rückgekoppelt, inwiefern sich dieses Handeln in ein quäkerspezifisches Deutungsschema einordnen ließ. ${ }^{70}$

Die folgende Studie versteht sich nicht als Organisationsgeschichte und doch steht eine Organisation im Mittelpunkt des Geschehens: das American Friends Service Committee. Von wem ist also die Rede, wenn im Folgenden vom AFSC gesprochen wird? Das Hauptinteresse gilt den Menschen, die das AFSC und seine Handlungen formten. Dabei geht es zunächst um diejenigen, die das AFSC im Laufe des Untersuchungszeitraums im Wesentlichen trugen: Mitglieder der Führungsebene und der Untersektionen des AFSC, freiwillige Helfer, die das Komitee auf Einsätze schickte, Mitglieder der Religious Society of Friends, die über Spendenkampagnen, Quäkerpublikationen und Informationsmaterial des AFSC mit dessen Arbeit in Berührung kamen und Anteil an ihm nahmen. Welche Erwartungen hatten sie an die Arbeit des Komitees? Welche Erfahrungen brachten sie dabei ein? Was glaubten sie? Welche politischen Ziele vertraten sie? Waren sie Internationalisten? Patrioten? Agenten des American Empire? Waren sie Revolutionäre oder Reformer? Waren sie von missionarischem Geist beseelt oder nüchterne Technokraten der Hilfe?

Bei der Beantwortung dieser Fragen zur Konstruktion der humanitären Identität des AFSC erscheinen Selbstzeugnisse und Selbstbeschreibungen der Quäker als wichtige, wenngleich allein nicht ausreichende Quellen. Dies vor allem, weil die Konstruktion dieser Identität sich ausnahmslos in einem dynamischen Wechselverhältnis mit der „Außenwelt“ vollzog. Hier findet ein weiterer Kreis von Stimmen Beachtung, welche die vorliegende Arbeit im Rahmen des

70 Zum Internationalismus der Quäker während der Zwischenkriegszeit liegen kaum Studien vor. Einführende Überlegungen bei Maureen Waugh, „Quakers, Peace and the League of Nations: The Role of Bertram Pickard,“ Quaker Studies 6, no. 1 (2001). 
Möglichen und des über die Quellenbestände Erreichbaren einzubeziehen versucht: Gemeint sind Sympathisanten und Mitstreiter der Quäker, die „friends of the Friends“, Verbündete und Kooperationspartner mit religiösem und säkularem Hintergrund, in Regierungsstellen und im Militär, in internationalen Organisationen; schließlich und nicht zuletzt auch lokale Helfer und die Empfänger der Hilfe: deutsche, russische, spanische Kinder, Frauen, Männer.

Die großen Hilfsoperationen des AFSC vor Beginn des Zweiten Weltkriegs bilden das Grundgerüst dieses Buches, in dessen Kapiteln jeweils spezifische Aspekte der Hilfe von übergeordneter Bedeutung beleuchtet werden. Nacheinander behandeln sie die Vorgeschichte (Kap. 1) und die Gründungsphase des AFSC mit dem Einsatz an den Fronten des Ersten Weltkriegs 1917 und 1918 (Kap. 2), die Kinderspeisungen in Deutschland von 1920 bis 1924 (Kap. 3) sowie die Hilfsaktion in Sowjetrussland (Kap. 4). Lediglich Kapitel 5, das sich im Kern dem Verhältnis von home und foreign service des AFSC am Übergang von den 1920er zu den 1930er Jahren widmet, weicht von dieser Systematik ab. Kapitel 6 und 7 behandeln in der Folge die großen Hilfseinsätze zugunsten der Opfer der Verfolgung in Nazi-Deutschland 1933 bis 1939 sowie abschließend im Spanischen Bürgerkrieg zwischen 1936 und 1939.

Den Auftakt bildet Kapitel 1, in dem die längere Geschichte humanitärer Aktivitäten der Quäker vor dem Hintergrund breiterer Entwicklungen innerhalb der Religious Society of Friends in den USA während des 19. Jahrhunderts bis zum Ersten Weltkrieg analysiert wird. Ins Bild rückt insbesondere die tiefe Verankerung der Quäker in breiteren Trends innerhalb des Protestantismus und das Verhältnis zwischen staatlicher Politik und privater Hilfe zu dieser Zeit.

Kapitel 2 beschreibt die Anfänge des AFSC im Ersten Weltkrieg als Teil eines globalen Moments in der Geschichte humanitärer Hilfe. Mit der Gründung einer Quäkerhilfsorganisation verbanden sich unterschiedliche Motive, die in der zugrunde liegenden Idee des „,constructive service“ ihren Ausdruck fanden: Es galt, wie zu zeigen sein wird, den Spagat zwischen patriotischem Bekenntnis einerseits und einem Hilfsangebot im Einklang mit den pazifistisch-religiösen Grundsätzen der Quäker andererseits zu bewerkstelligen, und dabei gleichzeitig als zentrale Stimme und Klammer einer alles andere als einheitlichen Glaubensgemeinschaft zu fungieren.

Im Mittelpunkt des dritten Kapitels stehen die „Quäkerspeisungen“ des AFSC in Deutschland nach dem Ende des Ersten Weltkriegs als erster großer Hilfseinsatz des Komitees in Friedenszeiten. Für das AFSC wurde der deutsche Einsatz, gleich auf mehrfache Weise zu einem Schlüsselereignis oder, in den Worten des AFSC selbst, einem „new Pennsylvania“: Nach dem Kriegseinsatz in Frankreich bedeutete Hilfe für die ehemaligen Feinde die Rückkehr zu einem authentischen „quakerly service“. Darüber hinaus spiegelte der deutsche Einsatz das Span- 
nungsfeld, in dem sich der „constructive service“ des AFSC auch nach Ende des Krieges bewegte: Ursprünglich aus der Zusammenarbeit mit den britischen Quäkern und im Kampf gegen die Blockade Deutschlands durch die Alliierten entstanden, übernahm das AFSC durch die Zusammenarbeit mit der American Relief Administration Herbert Hoovers eine Rolle als quasi-offizielle amerikanische Hilfsagentur - mit weitreichenden Folgen für das Verhältnis zu deutschen Empfängern und britischen Partnern und dadurch für das Selbstverständnis des Komitees.

Ganz ähnliche Fragen stellten sich den amerikanischen Quäkern im unmittelbar folgenden Hilfseinsatz des AFSC in Sowjetrussland zu Anfang der 1920er Jahre, der im Mittelpunkt des vierten Kapitels steht. Noch dringlicher als in Deutschland sollten sich die Quäker in Russland dem Dilemma ausgesetzt sehen, den Anspruch auf einen quäkerspezifischen Charakter ihres Einsatzes in Einklang mit den praktischen Anforderungen einer großangelegten Hilfsoperation zu bringen. In weitaus höherem Maße als in Deutschland löste der russische Einsatz sowohl innerhalb des Komitees als auch zwischen dem home office in Philadelphia und den Helfern vor Ort eine politische Debatte um die Position des Komitees aus.

Kapitel 5 taucht tief in die Debatten um die Zukunft des AFSC ein, die die Entwicklung des Komitees zu einer dauerhaften Quäkerhilfsorganisation während der Hilfseinsätze der frühen 1920er Jahre und darüber hinaus begleiteten. Ins Licht rücken dabei die Bemühungen des AFSC, zuweilen kollidierende Ansprüche miteinander zu versöhnen: als Service Committee mit einem deutlich erkennbaren sozialen Profil zu fungieren und gleichzeitig als Vertretung und Brücke für alle Richtungen des amerikanischen Quäkertums zu dienen. Den Hintergrund bildet sowohl das wachsende Engagement des AFSC als Hilfsagentur in den USA selbst, namentlich in Arbeitskonflikten, als auch der parallele Prozess der Annäherung an Staat und Regierung, die unter der Präsidentschaft Hoovers begann und im New Deal seinen Höhepunkt erreichte. Besonderes Augenmerk gilt dabei den vielfältigen Verbindungslinien und Wechselwirkungen, die zwischen dem neuen Arbeitsschwerpunkt in den USA und der weiterlaufenden auswärtigen Arbeit des foreign service verliefen. Den gemeinsamen Nenner all dieser Entwicklungen stellt dabei die Frage nach dem letzten Ziel und Sinn und mithin der message der Quäkerhilfe dar.

Kapitel 6 zeigt die zunächst zögerliche Rückkehr des AFSC in die Rolle einer international tätigen Hilfsorganisation vor dem Hintergrund der nationalsozialistischen Verfolgungen in Deutschland nach 1933. Zwei Aspekte stehen im Mittelpunkt: Zum einen beleuchtet es die enorme Bedeutung des Einsatzes für die Entwicklung des AFSC als Hilfsagentur, welche die Arbeit für die Verfolgten des Regimes in Deutschland, in den europäischen Zentren der Flucht sowie in den 
USA selbst zu koordinieren versuchte. Zum anderen werden im Kapitel Konturen eines quäkerspezifischen Hilfsansatzes deutlich, der im Angesicht des nationalsozialistischen Terrors bis an seine äußersten Grenzen geführt wurde.

Kapitel 7 knüpft in vieler Hinsicht unmittelbar an jenes über den deutschen Einsatz an. Der spanische Bürgerkrieg erscheint dabei als Katalysator einer Entwicklung, die die Etablierung des AFSC als Hilfsorganisation in einem Netz nationaler, internationaler und transnationaler Verbindungen zeigt. Dabei verdeutlicht der Einsatz des AFSC im spanischen Bürgerkrieg sowohl in seiner Praxis als auch in seiner Repräsentation in den USA die Vielschichtigkeit und Flexibilität des Neutralitätsanspruchs der Quäker, der hier gleichzeitig in neuer Weise als Mittel eingesetzt wurde, um sich auf dem „humanitären Markt“ zu behaupten. Schließlich erlaubt der Einsatz in einem politisch hochaufgeladenen Konflikt einen Einblick in die spezifische Mischung aus Annäherung und Abgrenzung, mit dem das AFSC sein Verhältnis zu verschiedenen Akteuren gestaltete, von der Regierung über den Kreis der politischen Solidaritätskomitees bis hin zum Völkerbund.

Kapitel 8 behandelt zum Abschluss den Zweiten Weltkrieg, der in vielen Bereichen eine Gelegenheit zur Bilanz bietet. Das AFSC erscheint darin als etablierte, national und international breit vernetzte Hilfsorganisation, in der sich die Entwicklung des humanitären Sektors ebenso wie die Spannungen des Humanitarismus während der Zwischenkriegszeit widerspiegeln. 